performance-based risk-sharing arrangement, multi-criteria decision analysis and economic evaluation.

\section{PP033 Patient And Public Involvement In Health Technology Assessment: The Brazilian Experience}

\section{AUTHORS:}

Tacila Mega, Aline Silva (aline.silveira@saude.gov.br), Clarice Petramale, Roberta Rabelo

\section{INTRODUCTION:}

The National Committee for Health Technology Incorporation (CONITEC) (1) was created in 2011, when the participation of civil society in the Health Technology Assessment (HTA) process was formalized in Brazil. According to legislation, patient and public involvement (PPI) in HTA occurs through: public consultations (PC); representation of SUS (Brazilian Public Health System) users in the plenary of CONITEC and by public hearings in relevant cases. Due the incipient culture of social participation in Brazil, strategies involving better communication, direct participation and popular education were developed to broaden and qualify this participation.

\section{METHODS:}

- Case study about PPI strategies developed in 5 years of CONITEC

- Analysis of documents and official records from the Brazilian Ministry of Health.

\section{RESULTS:}

Since its creation, the innovations of CONITEC regarding PPI were: creation of specific PC form to reproduce or represent the perspectives of patients and caregivers; summarized versions of technical reports written in a simplified language to improve users involvement; surveys prior to elaborating clinical guidelines, a bi-weekly educational program transmitted by streaming, and the recent launch of an HTA Users Guide and a mobile app.

After the implementation of these strategies (which started in 2014), there was an increase of annual contributions, from 2,584 in 2014 to 13,619 in 2015. Most participants were patients, family members or caregivers. Surveys concerning clinical guidelines received about 3,000 contributions. There were thirty-seven published society reports until December 2016. The publication of the HTA Users Guide and other related actions increased the number of accesses to the CONITEC website and its subsection for social participation. The educational program had more than 800 online accesses in five months.

\section{CONCLUSIONS:}

These actions allowed expanding and qualifying PPI beyond what is legally defined, and it is possible to predict an increasingly favorable scenario regarding the patient and public participation in HTA in Brazil.

\section{REFERENCES:}

1. National Committee for Health Technology Incorporation (CONITEC). Available in: www.conitec.gov.br.

\section{PP037 Quality Criteria And Good Practices In The Health Technology Assessment Spanish Network}

\section{AUTHORS:}

Sergio Márquez-Peláez

(sergio.marquez.ext@juntadeandalucia.es),Carmen Navarro-Palenzuela, Teresa Molina-Lopez, María del Mar Castellano-Zurera, José Luis Castro-Campos

\section{INTRODUCTION:}

The Spanish Network of Agencies for Health Technology Assessment (REDETS) is a group of eight agencies, units and services, depending on National and Regional Governments that coordinate their work within a 